\section{REVERSAL OF VALIDAMYCIN INHIBITION BY THE HYPHAL EXTRACT OF RHIZOCTONIA SOLANI}

Sir:

Validamycin (VM) showed no antimicrobial activity by the usual in vitro tests, although it inhibited the sheath blight of rice plants in vivo. VM also showed no inhibition of the initiation of the growth of Rhizoctonia solani, the causative agent of sheath blight of rice plants but it inhibited further extension of the hyphae, followed by an abnormal branching at the tips ${ }^{1}$. The inhibition of hyphal extension was more in the main hyphae than in the primary or secondary branches of $R$. solani. ${ }^{2}$ The remarkable prevention by VM of the sheath blight in vivo is now presumed to be due to the inhibition of the extension of main hyphae by abnormal branching. The mode of action of VM, however, has not yet been elucidated.

We have now found that an hyphal extract of $R$. solani antagonizes the action of VM, which may give a clue to elucidate the mode of action of VM.

The peripheral hyphae of a giant colony of $R$. solani, previously incubated on a potato sucrose plate at $28^{\circ} \mathrm{C}$ for 3 days, were initially used for the preparation of the hyphal extract. In later studies $R$. solani R-44 was grown in $200-\mathrm{ml}$ Erlenmeyer flasks containing $50 \mathrm{ml}$ of medium $\mathrm{S}$ (glucose $2 \%$, starch $3 \%$, corn steep liquor $1 \%$, soy bean flour $1 \%$, peptone $0.5 \%, \mathrm{NaCl} 0.3 \%$, $\mathrm{CaCO}_{3} 0.5 \%$ ). After 4-day incubation at $28^{\circ} \mathrm{C}$ on a rotary shaker, the hyphal cake was obtained by filtration. The hyphal cake (wet weight $118 \mathrm{~g}$ ) was extracted with 10 volumes $(\mathrm{v} / \mathrm{w})$ of $50 \%$ $\mathrm{MeOH}$ under reflux for 1 hour, and the extract was concentrated in vacuo to remove methanol. The resulting aqueous extract was passed through Amberlite IR-120 B column $\left(\mathrm{H}^{+}\right.$form, $3.5 \times$ $40 \mathrm{~cm})$ to remove the basic components. The effluent was further concentrated to a syrup and $2.2 \mathrm{~g}$ of the hyphal extract were obtained after drying in a silica gel desiccator.

The action of hyphal extract was examined by the modified dendroid test method, an assay method for $\mathrm{VM}^{3)}$. An agar disk (inoculum) of $R$. solani, which had previously been dipped in a 100 $\mu \mathrm{g} / \mathrm{ml}$ solution of $\mathrm{VM}$, was inoculated on a water agar plate. A cup containing $1 \%$ solution of the hyphal extract was also placed on the agar at the distance of $20 \mathrm{~mm}$ from the agar disk. The effect of hyphal extract on VM inhibition was examined during 6-day incubation. As shown in Fig. 1, the hyphal extension of $R$. solani was inhibited first after 2-day incubation, and then normal hyphal extension occurred gradually in a cup site. On the other hand, hyphal extension was inhibited even after 6-day incubation on the opposite site. This stimulation was observed only with the extract from apical hyphae but not with that from subapical hyphae. No stimulation of hyphal extension was observed in normal hyphae of $R$. solani, untreated with VM.

WAKAE reported that the formation of abnormal branching of $R$. solani was weak in the presence of inositol or fructose, that is, the reduction of pathogenicity of $R$. solani induced by VM (the

Fig. 1. Effect of the hyphal extract of Rhizoctonia solani on the action of validamycin.

A: Stimulation of hyphal extension by the hyphal extract from apical region.

B: No stimulation of hyphal extension by the hyphal extract from subapical region.

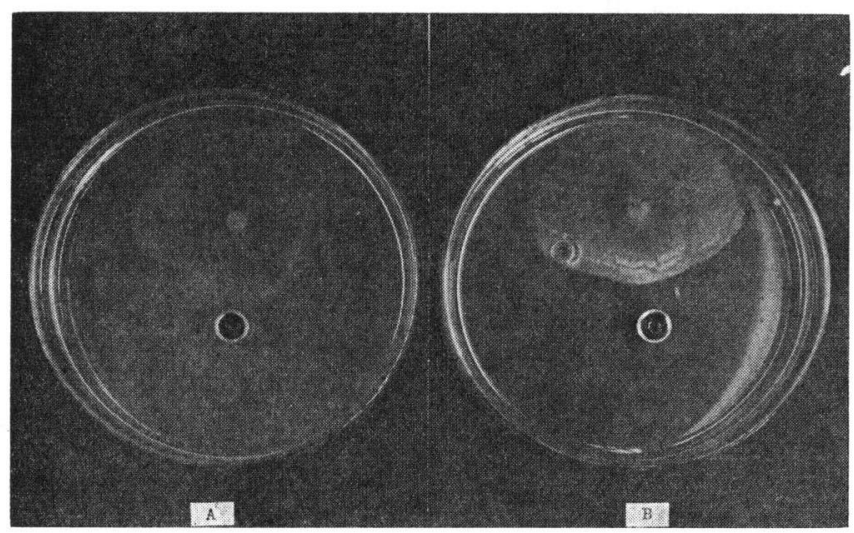


Fig. 2. Reversal of validamycin inhibition by the hyphal extract. Values represent the concentrations of the hyphal extract $(\mu \mathrm{g} / \mathrm{ml})$.

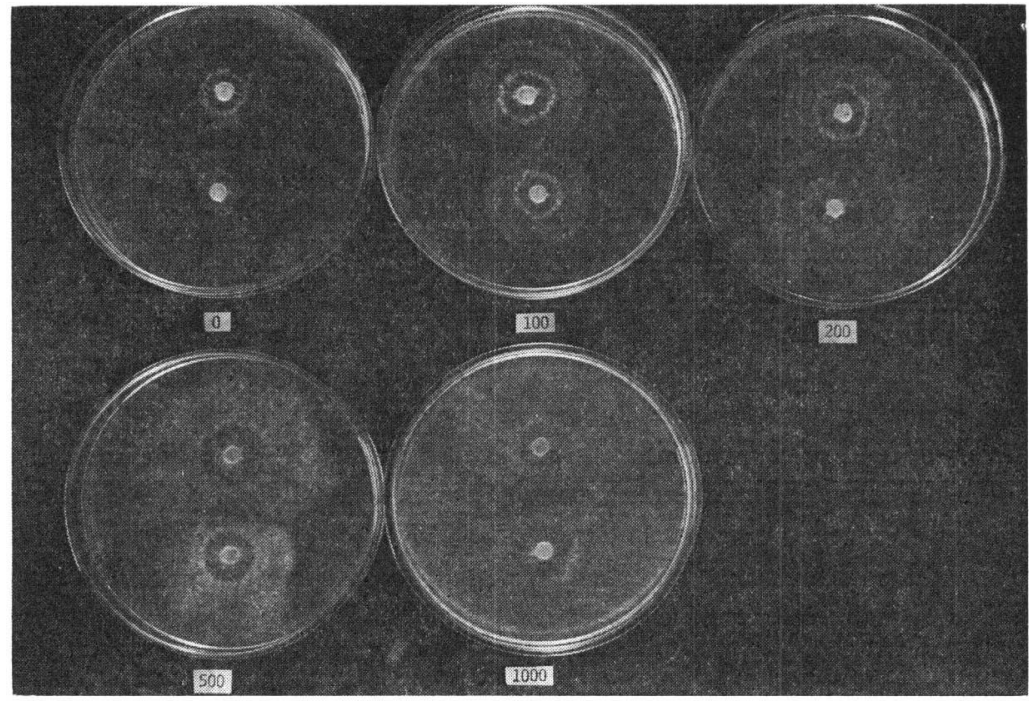

reduction of percent germination of cucumber seeds) was recovered in inositol to about one third, compared with the control (no VMtreatment) but not by fructose..$^{4,5)}$ The hyphal extract was presumed to contain various sugars and nitrogen-containing compounds which affect VM inhibition. The affect of these compounds on VM inhibition was examined by the method described above. The extension of the hyphae previously inhibited by VM was slightly stimulated by glucose, fructose or peptone but not by inositol, while a similar stimulation was also observed in the normal hyphae, untreated with VM. The stimulation by these compounds was considered to be due to merely an enrichment of medium, because the effect was similarly observed both in the normal hyphae and in the VMtreated hyphae. On the other hand, the hyphal extract stimulated only the extension of the VMtreated hyphae and not that of the normal hyphae. Thus, a significant stimulation by the hyphal extract was considered to be different from that of these compounds.

An agar disk of $R$. solani, previously treated with $10 \mu \mathrm{g} / \mathrm{ml}$ solution of VM was inoculated on a water agar plate containing various concentrations of the hyphal extract. An antagonistic action of hyphal extract against VM was examined and the results are shown in Fig. 2. The hyphal extension of $R$. solani was inhibited by
Table 1. Reversal of validamycin inhibition by the hyphal extract.

\begin{tabular}{r|r|r|r|r}
\hline \multirow{2}{*}{$\begin{array}{c}\text { Hyphal extract } \\
(\mu \mathrm{g} / \mathrm{ml})\end{array}$} & \multicolumn{4}{|c}{ Growth zone diameter $(\mathrm{mm})$} \\
\cline { 2 - 5 } & 1 day & 2 day & 3 day & 4 day \\
\hline 0 & 16.0 & 29.0 & 38.0 & 47.7 \\
500 & 18.7 & 30.3 & 39.3 & 50.5 \\
1,000 & 19.7 & 34.0 & 46.7 & 64.5 \\
2,000 & 21.7 & 55.0 & 82.3 & $>90.0$ \\
5,000 & 21.0 & 69.7 & $>90.0$ & $>90.0$ \\
10,000 & 26.0 & 76.0 & $>90.0$ & $>90.0$ \\
\hline
\end{tabular}

Ten $\mu \mathrm{g} / \mathrm{ml}$ of validamycin was used.

VM in the absence of the hyphal extract. This VM inhibition was antagonized by the addition of the hyphal extract and the hyphal extension was increased in response to the increased concentration of the hyphal extract. Table 1 shows the time course change of antagonistic action of the hyphal extract against VM in another experiment. These findings suggest that a factor which is concerned with hyphal extension exists in apical hyphae and VM affects the action of the factor. Details of the antagonistic action of the hyphal extract will be reported in the next paper.

\section{Acknowledgement}

We wish to thank Takeda Chemical Industries, Ltd. for the supply of validamycin. 


\section{Motoo Shibata \\ MASARU Uyeda \\ KAZUO MORI}

Laboratory of Medicinal Microbiology, Faculty of Pharmaceutical Sciences,

Kumamoto University, Kumamoto, Japan

(Received February 7, 1980)

\section{References}

1) Iwasa, T.; E. Higashide, H. Yamamoto \& M. ShIBATA: Studies on validamycins, new antibiotics. II. Production and biological properties of validamycins A and B. J. Antibiotics 24: $107 \sim 113,1971$
2) Nioh, T. \& S. Mizushima: Effect of validamycin on the growth and morphology of Pellicularia sasakii. J. Gen. Appl. Microbiol. 20: $373 \sim 383,1974$

3) Iwasa, T.; E. Higashide \& M. Shibata: Studies on validamycins, new antibiotics. III. Bioassay methods for the determination of validamycin. J. Antibiotics 24: 114 118, 1971

4) WaKae, O. \& K. MatsuUra: Validamycin, a new antibiotic for Rhizoctonia disease control. Proceed. 1st Internat. Congr. Internat. Ass. Microbiol. Soc. 3: 620 629, 1975

5) WaKae, O. \& K. MatsuUra: Studies on the control effect of validamycin on sheath blight of rice plant. XI. Action mechanism (1). Ann. Phytopath. Soc. Japan 40: 224, 1974 